\title{
A NOTE ABOUT THE DERIVATIVES OF LEGENDRE POLYNOMIALS
}

\author{
N. DU PLESSIS
}

Here is an alternative, shorter, proof of the result proved by Grosswald. ${ }^{1}$

We have

$$
\left(1-2 x \rho+\rho^{2}\right)^{-1 / 2}=\sum_{0}^{\infty} P_{n}(x) \rho^{n} .
$$

Differentiating $r$ times with respect to $x$ and putting $x=1$, we have

$$
1 \cdot 3 \cdot s \cdot \cdots(2 r-1) \rho^{r}(1-\rho)^{-2 r-1}=\sum_{0}^{\infty} P_{n}^{(r)}(1) \rho^{n} .
$$

Equating coefficients of $\rho^{n}$ we now have

$$
P_{n}^{(r)}(1)=\frac{(n+r) !}{2^{r} r !(n-r) !} .
$$

Similarly, we may show that

$$
P_{n}^{(r)}(-1)=(-1)^{n+r} \frac{(n+r) !}{2 r r !(n-r) !} .
$$

Rhodes University College

Received by the editors, January 15, 1951.

1 E. Grosswald, On a simple property of Legendre's polynomials, Proceedings of the American Mathematical Society vol. 1 (1950) pp. 553-554. 